\title{
Stability Improvement of Motion of a Rotor With an Active Control Method
}

\author{
M. F. LIAO \\ Institute of Monitoring and Control for Rotating Machinery and Windtubines (NPU \& TU Berlin), \\ Northwestern Polytechnical University(NPU), Xian 710072, China \\ R. GASCH \\ O. KOLK \\ T. KLETSCHKOWSKI \\ Technical University of Berlin. Germany
}

(Received 2I April 1997; accepted 10 Augusl 1998)

Abstract: The so-called phase-locked delayed feedback control method is applicd to a Jeffcott rotor supported by rolling-clement bearings but with negative damping and then to the same rotor supported by joumal bearings, to improve the stability of motion of the rotor. The control parameters are determined in terms of the characteristic equation of the controlled rotor. The results of numerical simulations show that the stability of motion of the rotor is greatly improved with the help of the phase-locked delayed feedback control method.

Key Words: Phase-locked delayed fecdback control, stability improvement, rotor

\section{INTRODUCTION}

The stability of motion of a rotor is a major concern in the practice. The change in oil temperature, rub between rotor and stator or some other disturbances-for example, earthquake-and fluctuations in the net voltage can bring a rotor into unstable motion. Although much effort is exerted to cope with the instability, the unstable vibration cannot be completely avoided. It causes serious damage to a machine when it occurs and is not eliminated at once. Hence, it is desirable to suppress the instability of a rotor when disturbances appear.

The vibration of a rotor excited by imbalance is periodic. It should be suppressed by balancing the rotor, but not by a control system. Otherwise, strong forces will be transmitted to the foundation, on one hand, and, on the other hand, the control system must supply much power, which gives rise to the difficulties in the realization of the control system.

To this end, an active control method is desired, which is only activated when the motion of a rotor tends to be unstable but does not interfere with the periodic motion resulting from imbalance. In this way, both the unstable vibration of the rotor and the forces transmitted to the foundation will be reduced. 
In this work, the so-called phase-locked delayed feedback control method (Faragher, 1996; Gasch and Liao, 1996; Krodkiewski and Faragher, 1995) is applied to a Jefficott rotor supported by rolling-element bearings but with negative damping and to the same rotor supported by journal bearings. The control parameters are acquired from the characteristic equation. The results of numerical simulations show that the stability of motion of a rotor is greatly improved with the help of the phase-locked delayed feedback control method.

\section{STABILITY IMPROVEMENT OF MOTION OF A JEFFCOTT ROTOR SUPPORTED BY ROLLING-ELEMENT BEARINGS WITH THE HELP OF THE PHASE-LOCKED DELAYED FEEDBACK CONTROL}

To gain an insight into the phase-locked delayed feedback control method, we first consider a Jeffcott rotor supported by ball bearings, but with negative damping.

Figure 1 (a) indicates a Jeffcott rotor supported on ball bearings, and Figure 1 (b) shows a block diagram of the phase-locked delayed feedback control system. The differential equations depicting the motion of the rotor are

$$
\begin{aligned}
& m \ddot{x}+c \dot{x}+s x=m \varepsilon \Omega^{2} \cos \Omega t+A[x(t)-x(t-T)]+B[\dot{x}(t)-\dot{x}(t-T)] \\
& m \ddot{y}+c \dot{y}+s y=m \varepsilon \Omega^{2} \sin \Omega t+A[y(t)-y(t-T)]+B[\dot{v}(t)-\dot{y}(t-T)]
\end{aligned}
$$

where $m$ represents the mass of the rotor; $c$ and $s$ the damping and stiffness, respectively; $A$ and $B$ are the control parameters; and $T\left(=\frac{2 \pi}{\Omega}\right)$ is the period of the excitation due to imbalance.

Arranging equations ( 1 ) and (2) in complex form, we obtain

$$
\ddot{z}+2 D \omega \dot{z}+\omega^{2} z=\varepsilon \Omega^{2} e^{j \Omega t}+a[z(t)-z(t-T)]+b[\dot{z}(t)-\dot{z}(t-T)],
$$

where $z=x+j y$

$$
\omega=\sqrt{\frac{s}{m}}, \quad D=\frac{c}{2 \sqrt{s \cdot m}}, \quad a=\frac{A}{m}, \quad b=\frac{B}{m} .
$$

Equation (3) has one periodic particular solution that does not depend on the control parameters $a$ and $b$ :

$$
\begin{aligned}
& z(t)=z(t+T)=Z e^{j \Omega 2 t} \\
& Z=\frac{\varepsilon \Omega^{2}}{\omega^{2}-\Omega^{2}+2 j D \omega \Omega} .
\end{aligned}
$$



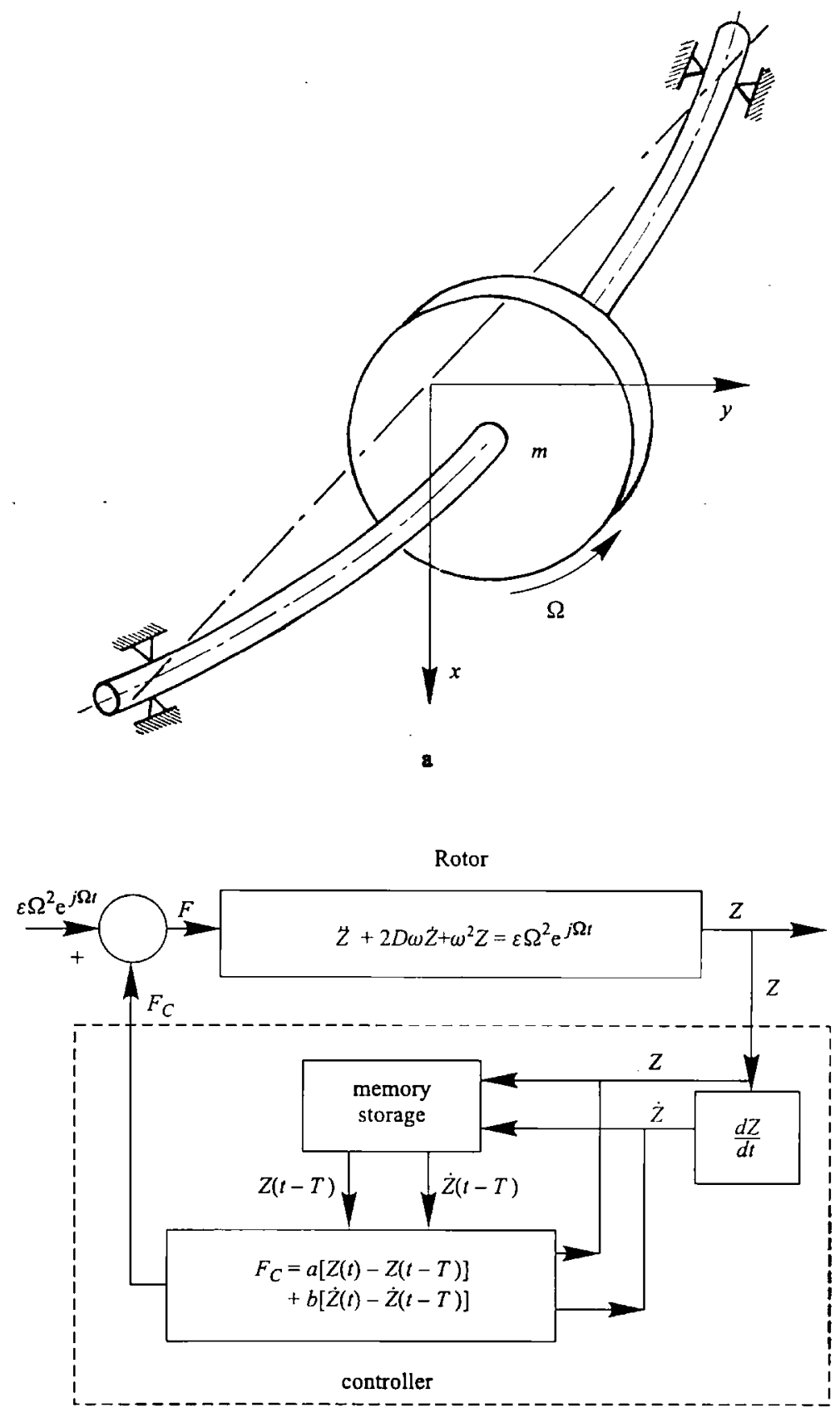

b

Figure 1. (a) a rotor supported on rolling-element bearings and (b) the control system. 


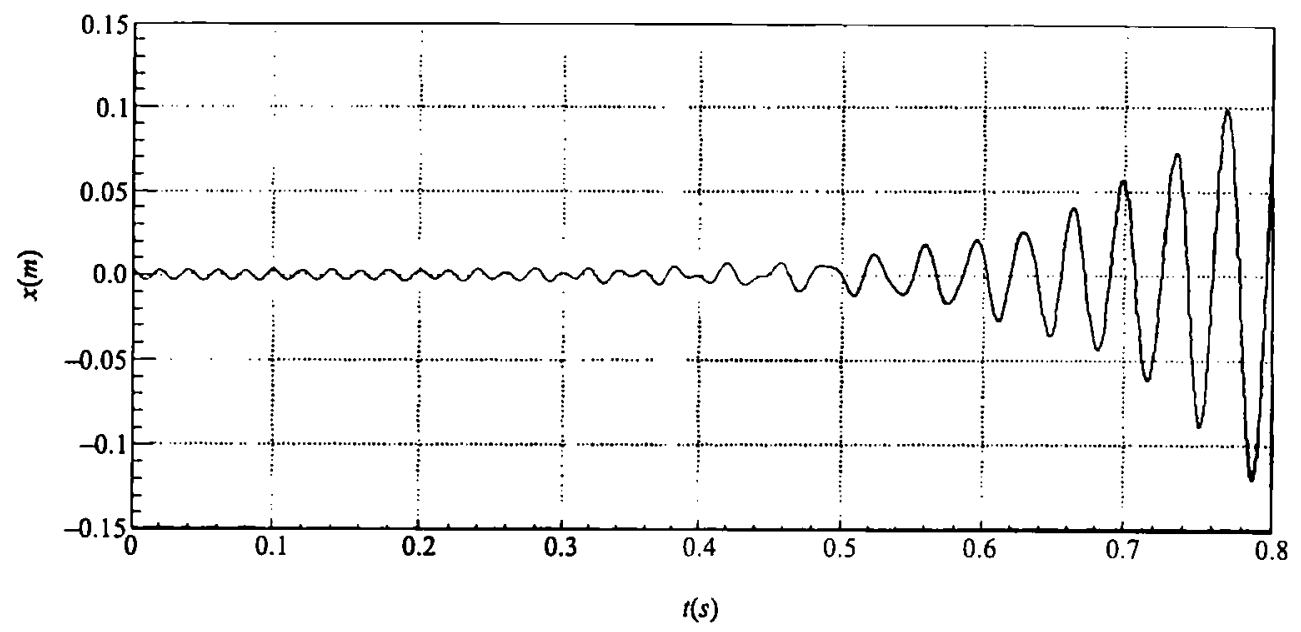

a

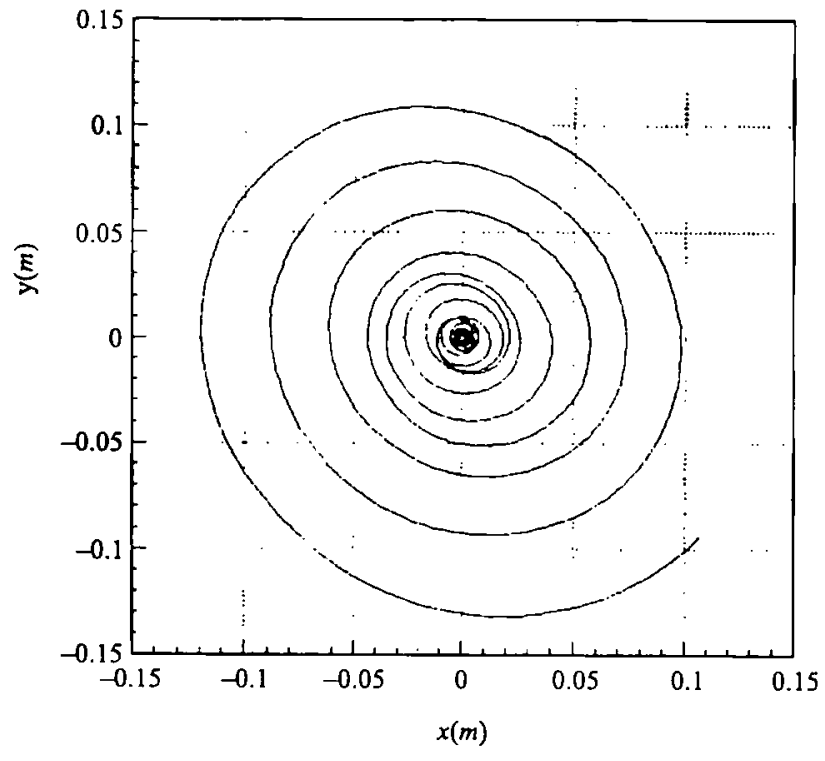

b

Figure 2. The unstable motion of a rotor with negative damping. (a) the unstable vibration in direction $x$, and (b) the unstable orbit of the rotor. 
Table 1. Relationship of control parameters $a$ and $b$ to the real and imaginary parts, $R$ and $U$, of the dominant root.

\begin{tabular}{cccc}
\hline$a$ & $b$ & $R$ & $U$ \\
\hline 0 & -41.7 & -0.03168 & \pm 194.88 \\
0 & -45.0 & -2.93444 & \pm 198.37 \\
0 & -50.0 & -6.29627 & \pm 204.38 \\
\hline
\end{tabular}

The periodic solution (equation (4)) is identical with that of the uncontrolled system. This is one of the most important features of the phase-locked delayed feedback control method.

When no control is present, that is, $a=b=0$, the homogeneous solution of equation (3) takes the form:

$$
z_{0}=e^{-\omega D t}\left(Z_{10} e^{j \sqrt{1-D^{2}} \omega t}+Z_{20} e^{-j \sqrt{1-D^{2}} \omega t}\right)
$$

From equation (6), it follows that the periodic motion of the uncontrolled rotor system depicted by equation (4) is unstable; when the damping $D$ is negative, that is, if a disturbance takes place, the disturbed motion grows to infinity with time. Figures 2 (a) and (b) show the unstable motion attributed to a negative damping (damping $D=-5 \%$ ). A negative damping can result from inner damping of material, seal, rub between rotor and stator, or other abnormal sources. Hence, even such a simple rotor may be also involved in an unstable motion.

To keep the rotor system in stable motion, the control is now applied to it. We deal with only the homogeneous equation of equation (3), which governs the stability of the solution (equation (4)), that is,

$$
\ddot{z}+2 D \omega \dot{z}+\omega^{2} z=a[z(t)-z(t-T)]+b[\dot{z}(t)-\dot{z}(t-T)] .
$$

The homogeneous solution to equation (7) with control parameters can be assumed to have the form

$$
z_{0}=Z_{0} e^{i \prime}
$$

Substituting it into equation (7), we obtain the characteristic equation

$$
\lambda^{2}+2 D \lambda \omega+\omega^{2}-(a+\lambda b)\left(1-e^{-i T}\right)=0 .
$$

With the following assumption

$$
\lambda=R+j U
$$

equation (9) can be separated into the real and imaginary parts

$$
R^{2}-U^{2}+\omega^{2}-(a+R b)\left(1-e^{-R T} \cos U T\right)+2 R D \omega+U b e^{-R T} \sin U T=0
$$




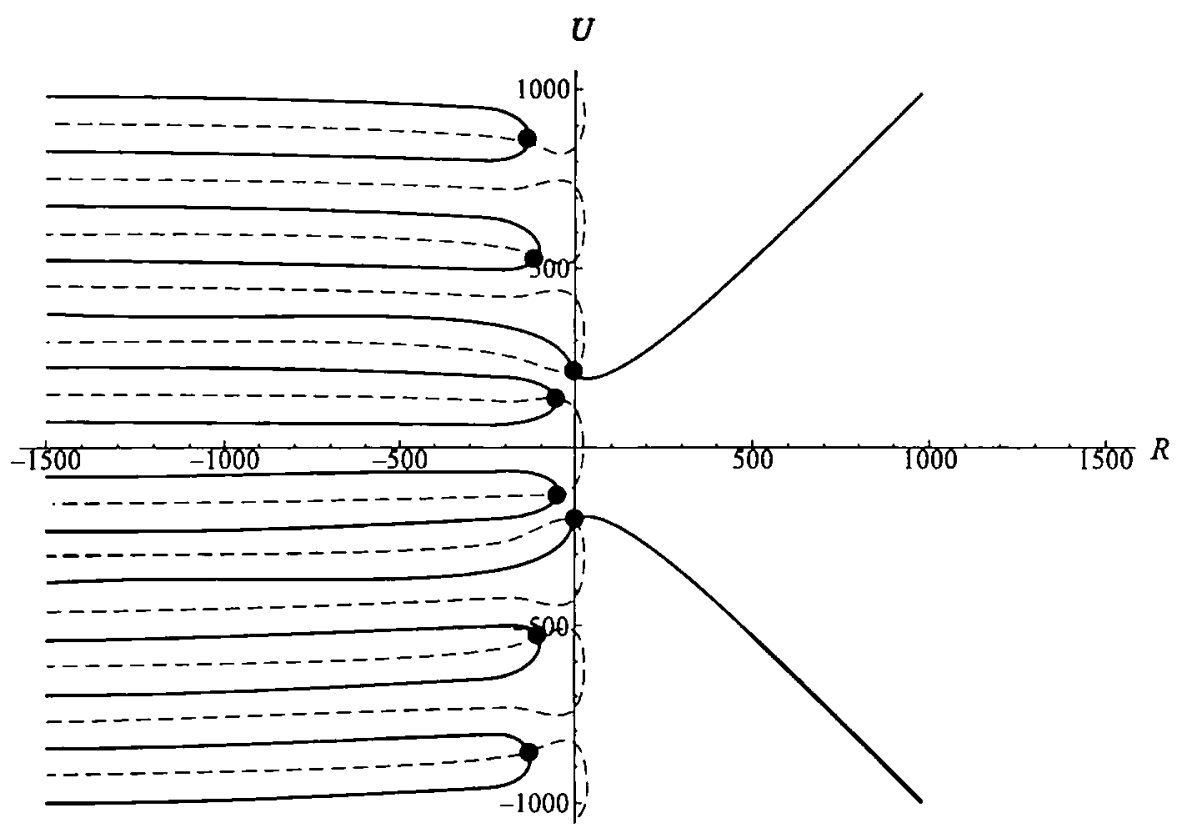

Figure 3. The real and imaginary parts of the roots of the characteristic equations (11) and (12) with $a=0$ and $b=-41.7$. The solid lines are the solution to equation(11); the dashed lines are the solution to equation (12); the intersection points are the solution to equations (11) and (12).

$$
2 R U+2 U D \omega-U b\left(1-e^{-R T} \cos U T\right)-(a+R b) e^{-R T} \sin U T=0 .
$$

The values of $R$ and $U$, which simultaneously satisfy both equations (11 and 12), define the roots of the characteristic equation. Clearly, the homogeneous solution (equation (8)) will decay with time $t$ if the value of the real part, $R$, is negative. We define the root of equation (9), which has the largest real part $R$, as the dominant root. If the dominant root has negative real part, then all the roots have negative real parts. Thus, we choose proper control parameters $a$ and $b$, so that all the roots of the characteristic equations (11) and (12) have negative real parts, then the homogeneous solution due to a disturbance will decrease with time and the periodic motion (equation (4)) will become asymptotically stable. With the help of a computer program, equations (11) and (12) can be solved and the values of $R$ and $U$, which satisfy both equations (11) and (12), can be found.

As an example, the following system parameters are given:

$$
\omega=1801 / s, \varepsilon=0.002 m, T=0.02 s, D=-0.2 .
$$

In terms of both equations (11) and (12), the following control parameters $a$ and $b$ are chosen,

$$
a=0, b=-41.71 / s,
$$




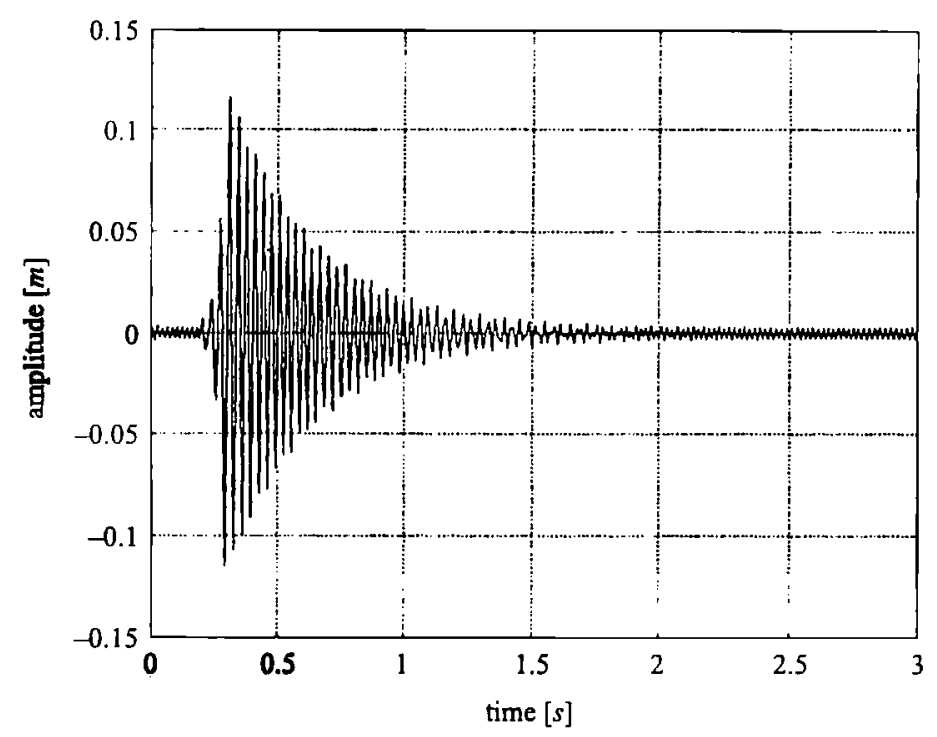

Figure 4. The stabilization of unstable vibration of a rotor with negative damping.

which make all the real parts of the eigenvalues negative. Hence, the rotor becomes stable. Figure 3 shows a plot of the solution for $R$ and $U$ in terms of equations (11) and (12). The points where the lines intersect, that is, where both equations (11) and (12) are satisfied, are the characteristic roots of equation (9). Table 1 gives the relationship of the control parameters $a$ and $b$ to the real and imaginary parts, $R$ and $U$, of the dominant root.

To simulate an abnormal operation process of a rotor, a positive damping $(D=0.2)$ is first introduced in the rotor. The homogeneous solution decays and the rotor vibrates with a constant amplitude and at the frequency of imbalance. At time $t_{0}=0.2 \mathrm{~s}$, the damping becomes negative $(D=-0.2)$, the homogeneous solution due to a disturbance grows with time, and the motion of the rotor becomes unstable. At $t_{1}=0.24 \mathrm{~s}$, the control parameters $a$ and $b$ are introduced in the system, then the homogeneous solution decreases with time quickly and the rotor retums to the periodic motion excited by imbalance. Figure 4 shows the stabilization of the unstable motion. The periodic motion of the controlled rotor is identical with that of the uncontrolled rotor. It shows that the phase-locked delayed feedback control method does not change the periodic motion of the uncontrolled rotor, while it improves its stability.

\section{STABILITY IMPROVEMENT OF MOTION OF A JEFFCOTT ROTOR SUPPORTED BY JOURNAL BEARINGS WITH THE HELP OF THE PHASE- LOCKED DELAYED FEEDBACK CONTROL}

Journal bearings can bring high damping in a rotor, which suppresses the vibration due to imbalance of the rotor particularly at critical speeds. However, journal bearings can cause 


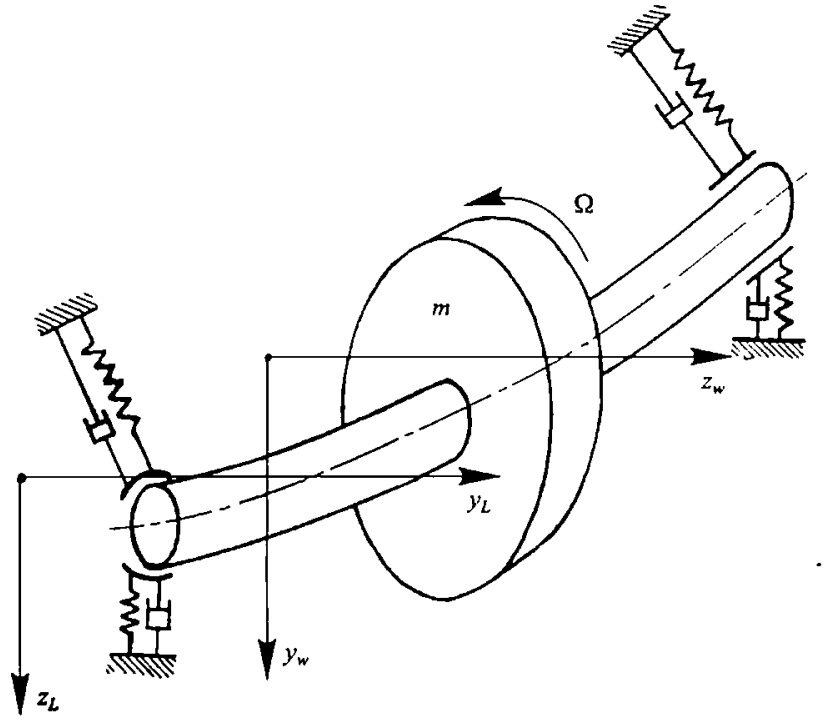

Figure 5. A Jeffcott rotor supported by journal bearings.

an unstable vibration of a rotor through a phenomenon known as oil whip, which is a selfexcited vibration induced by the oil film (Someya et al., 1989). In this case, the rotor vibrates violently. If it is not eliminated immediately, bearing failure and damage to the rotor may result.

To this end, the phase-locked delayed feedback control method is applied to a Jeffcott rotor supported by journal bearings, shown in Figure 5, to improve the stability of the rotor system.

\subsection{Differential Equations of Motion of the Controlled Rotor Supported by Journal Bearings}

The differential equation of motion of the rotor can be expressed in the matrix form

$$
\mathbf{M} \ddot{x}(t)+\mathbf{D} \dot{x}(t)+\mathbf{S} x(t)=F_{\text {imbalance }}+F_{\text {control }}[x(t), x(t-T), \dot{x}(t), \dot{x}(t-T)],
$$

where

$$
x=\left[\begin{array}{llll}
z_{r} & y_{r} & z_{b} & y_{b}
\end{array}\right]^{T}
$$

is the displacement vector of the rotor and bearing,

$$
\mathbf{M}=\left[\begin{array}{cccc}
m & 0 & 0 & 0 \\
0 & m & 0 & 0 \\
0 & 0 & 0 & 0 \\
0 & 0 & 0 & 0
\end{array}\right]
$$


is the mass matrix, and

$$
\begin{gathered}
\mathbf{D}=\left[\begin{array}{cccc}
0 & 0 & 0 & 0 \\
0 & 0 & 0 & 0 \\
0 & 0 & d_{z=} & d_{z y} \\
0 & 0 & d_{y z} & d_{y y}
\end{array}\right] \\
\mathbf{S}=\left[\begin{array}{cccc}
s & 0 & -s & 0 \\
0 & s & 0 & -s \\
-\frac{1}{2} s & 0 & s_{z:}+\frac{1}{2} s & s_{z y} \\
0 & -\frac{1}{2} s & s_{y z} & s_{y y}+\frac{1}{2} s
\end{array}\right]
\end{gathered}
$$

are the damping and stiffness matrices, respectively, which are related to the Sommerfeld number of the journal bearings (Gasch and Pfuetzner, 1975).

The right vectors of equation (13) represent the excitation due to imbalance and the control forces, respectively, that is,

$$
\begin{aligned}
& F_{\text {imbalance }}=\varepsilon m \Omega^{2}\left[\begin{array}{llll}
\cos \Omega t & \sin \Omega t & 0 & 0
\end{array}\right]^{T} \\
& F_{\text {control }}=\left[\begin{array}{c}
A_{z}\left(z_{r}(t)-z_{r}(t-T)\right)+B_{z}\left(\dot{z}_{r}(t)-\dot{z}_{r}(t-T)\right) \\
A_{y}\left(y_{r}(t)-y_{r}(t-T)\right)+B_{y}\left(\dot{y}_{r}(t)-\dot{y}_{r}(t-T)\right) \\
0 \\
0
\end{array}\right],
\end{aligned}
$$

where $A_{z}, B_{z}, A_{y}$, and $B_{y}$ represent the control parameters in $z$ - and $y$-directions, respectively.

The particular periodic solution to equation (13) should be identical with that of the uncontrolled rotor system $\left(A_{z}=B_{z}=A_{y}=B_{y}=0\right)$.

\subsection{Equations of Perturbations}

To gain an insight into the stability of the controlled rotor, the perturbated motion of the rotor is introduced as follows

$$
\underline{\tilde{x}}=\underline{x}_{0}+\Delta x,
$$

where $\underline{x}_{0}$ is the periodic motion and $\Delta \underline{x}$ is the perturbations. 
Substituting equation(16) into equation (13), we obtain the perturbed equation

$$
\begin{aligned}
& \mathrm{M} \Delta \ddot{x}+\mathbf{D} \Delta \dot{x}+\mathbf{S} \Delta x \\
& =\left\{\begin{array}{c}
A_{z}\left[\Delta z_{r}(t)-\Delta z_{r}(t-T)\right]+B=\left[\Delta \dot{z}_{r}(t)-\Delta \dot{z}_{r}(t-T)\right] \\
A_{y}\left[\Delta y_{r}(t)-\Delta y_{r}(t-T)\right]+B_{y}\left[\Delta \dot{y}_{r}(t)-\Delta \dot{y}_{r}(t-T)\right] \\
0 \\
0
\end{array}\right\} .
\end{aligned}
$$

The solution can be given by

$$
\Delta x=\Delta \hat{x} e^{\lambda t}=\left[\begin{array}{llll}
\Delta \hat{z}_{r} & \Delta \hat{y}_{r} & \Delta \hat{z}_{b} & \Delta \hat{y}_{b}
\end{array}\right]^{T} e^{\lambda l} .
$$

Inserting it into equation(17), we obtain the characteristic equation

$$
\operatorname{det}\left[\begin{array}{cc}
\lambda^{2} m+s-\left(A_{z}+\lambda B_{z}\right)\left(1-e^{-i T}\right) & 0 \\
0 & \lambda^{2} m+s-\left(A_{y}+\lambda B_{y}\right)\left(1-e^{-i T}\right) \\
-\frac{1}{2} s & 0 \\
0 & -\frac{1}{2} s \\
-s & 0 \\
0 & -s \\
\lambda d_{z=}+s_{z=}+\frac{1}{2} s & \lambda d_{z y}+s_{z y} \\
\lambda d_{y z}+s_{y z} & \lambda d_{y y}+s_{y y}+\frac{1}{2} s
\end{array}\right]=0 .
$$

Dividing the first two rows of determinant (19) by $s$ and multiplying the last two rows by $\frac{S_{0} \Delta R}{F_{\text {stat }}}$, we obtain the following dimensionless characteristic equation:

$$
\operatorname{det}\left[\begin{array}{cc}
\frac{\lambda^{2}}{\omega^{2}}+1-\left(a_{z}+\frac{\lambda}{\omega} b_{z}\right)\left(1-e^{-\lambda T}\right) & 0 \\
0 & \frac{\lambda^{2}}{\omega^{2}}+1-\left(a_{y}+\frac{\lambda}{\omega} b_{y}\right)\left(1-e^{-i T}\right) \\
-\frac{S_{0}}{\mu} & 0 \\
0 & -\frac{S_{0}}{\mu}
\end{array}\right.
$$




$$
\left.\begin{array}{cc}
-1 & 0 \\
0 & -1 \\
\frac{\lambda}{\Omega} \beta_{z=}+\gamma_{z=}+\frac{S_{0}}{\mu} & \frac{\lambda}{\Omega} \beta_{z y}+\gamma_{z y} \\
\frac{\lambda}{\Omega} \beta_{y z}+\gamma_{y=} & \frac{\lambda}{\Omega} \beta_{y y}+\gamma_{y y}+\frac{S_{0}}{\mu}
\end{array}\right]=0,
$$

where

$$
a_{z}=\frac{A_{z}}{s}, \quad b_{z}=\frac{B_{z}}{\omega m}, \quad a_{y}=\frac{A_{y}}{s}, \quad b_{y}=\frac{B_{y}}{\omega m},
$$

$\omega=\sqrt{\frac{s}{m}}$ is the eigenfrequency of the rotor supported by ball bearings

$S_{0}=\frac{F_{\text {stat }} \psi^{2}}{2 W R \eta_{\mathrm{oil}} \Omega}$ is the Sommerfeld number (Gasch and Pfuetzner, 1975)

$\beta_{i k}=d_{i k} \frac{S_{0} \Delta R \Omega}{F_{\text {st:at }}}$ the dimensionless damping coefficients of the journal bearing $(i, k=z, y)$

(Gasch and Pfuetzner, 1975)

$\gamma_{i k}=s_{i k} \frac{S_{0} \Delta R}{F_{\text {stat }}}$ is the dimensionless stiffness coefficients of the journal bearing $(i, k=z, y)$

(Gasch and Pfuetzner, 1975)

$\mu=\frac{f}{\Delta R}=\frac{m g / s}{R-r}$ is the ratio of the static deflection to the bearing clearance,

with

$F_{\text {st: }: 1}=\frac{m g}{2}$ being the static bearing load

$W=$ bearing width

$R=$ bearing radius

$r=$ journal radius

$\psi=\frac{R-r}{r}$ is the relative clearance

$\Omega=$ angular velocity of the journal

$\eta_{\mathrm{oil}}=$ viscosity of oil film.

\subsection{Stability Margin of Motion of the Uncontrolled Rotor}

If no control acts on the rotor, that is, $a_{z}=b_{z}=a_{y}=b_{y}=0$, the characteristic equation (20) can be arranged into a polynomial of degree 6 with respect to unknown $\left(\frac{\lambda}{\omega}\right)$ :

$$
r_{6}\left(\frac{\lambda}{\omega}\right)^{6}+r_{5}\left(\frac{\lambda}{\omega}\right)^{5}+r_{1}\left(\frac{\lambda}{\omega}\right)^{4}+r_{3}\left(\frac{\lambda}{\omega}\right)^{3}+r_{2}\left(\frac{\lambda}{\omega}\right)^{2}+r_{1}\left(\frac{\lambda}{\omega}\right)+r_{0}=0 .
$$


The coefficients here are given by

$$
\begin{aligned}
& r_{0}=A_{0} \\
& r_{1}=A_{1}\left(\frac{\omega}{\Omega}\right) \\
& r_{2}=2 A_{0}+\left(\frac{S_{0}}{\mu}\right) A_{4}+\left(\frac{\omega}{\Omega}\right)^{2} A_{2} \\
& r_{3}=\left(\frac{\omega}{\Omega}\right)\left(2 A_{1}+\frac{S_{0}}{\mu} A_{3}\right) \\
& r_{4}=2\left(\frac{\omega}{\Omega}\right)^{2} A_{2}+A_{0}+\left(\frac{S_{0}}{\mu}\right) A_{4}+\left(\frac{S_{0}}{\mu}\right)^{2} \\
& r_{5}=\left(\frac{\omega}{\Omega}\right)^{2}\left(A_{1}+\frac{S_{0}}{\mu} A_{3}\right) \\
& r_{6}=\left(\frac{\omega}{\Omega}\right)^{2} A_{2},
\end{aligned}
$$

with the following notations

$$
\begin{aligned}
& A_{0}=\gamma_{z=} \gamma_{y y}-\gamma_{z y} \gamma_{y=} \\
& A_{1}=\beta_{z=} \gamma_{y y}+\beta_{y y} \gamma_{z=}-\left(\beta_{z y} \gamma_{y z}+\beta_{y=} \gamma_{z y}\right) \\
& A_{2}=\beta_{z=} \beta_{y y}-\beta_{y=} \beta_{z y} \\
& A_{3}=\beta_{z=}+\beta_{y y} \\
& A_{4}=\gamma_{z=}+\gamma_{y y} .
\end{aligned}
$$

Introducing the stability margin

$$
\lambda=j \omega_{\lim }
$$

into equation (21) and separating the real and imaginary parts of the equation give the following two equations

$$
-r_{6}\left(\frac{\omega_{\lim }}{\omega}\right)^{6}+r_{4}\left(\frac{\omega_{\lim }}{\omega}\right)^{4}-r_{2}\left(\frac{\omega_{\lim }}{\omega}\right)^{2}+r_{0}=0
$$




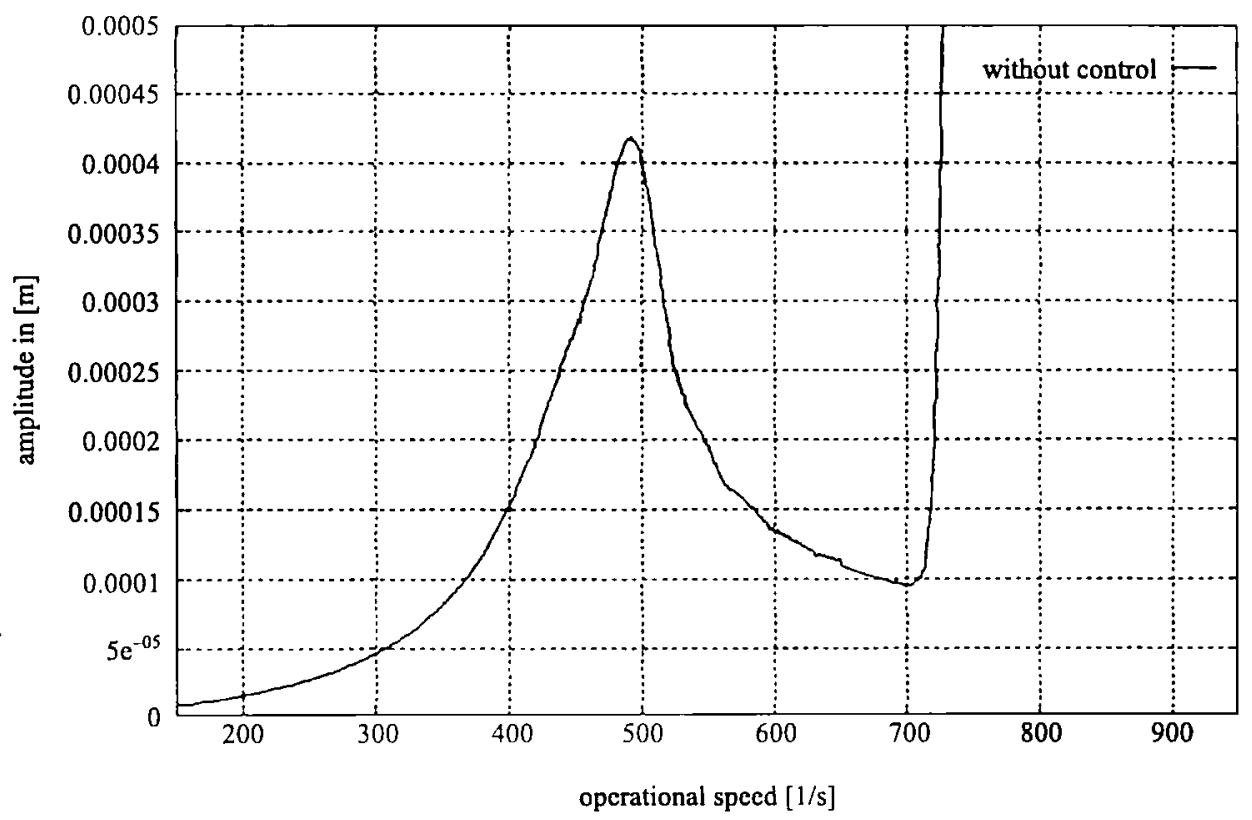

Figure 6. Response amplitude to imbalance versus operational speed.

$$
r_{5}\left(\frac{\omega_{\lim }}{\omega}\right)^{5}-r_{3}\left(\frac{\omega_{\lim }}{\omega}\right)^{3}+r_{1}\left(\frac{\omega_{\lim }}{\omega}\right)=0 .
$$

In terms of the two equations, we can determine the frequency with which the rotor vibrates in the stability boundary

$$
\left(\frac{\omega_{\lim }}{\omega}\right)^{2}=\frac{1}{1+\frac{S_{0}}{\mu} \frac{A_{3}}{A_{1}}}
$$

and furthermore obtain the stability margin

$$
\left(\frac{\Omega_{\lim }}{\omega}\right)^{2}=\frac{A_{2} A_{3}^{2}}{1+\frac{S_{0}}{\mu} \frac{A_{3}}{A_{1}}} \cdot \frac{1}{A_{1}^{2}-A_{1} A_{3} A_{4}+A_{0} A_{3}^{2}},
$$

which means that the rotor will become unstable when the operational speed exceeds the stability margin, equation (28).

The amplitude of the response to imbalance excitation is illustrated in Figure 6 . It is determined by direct integration of equation (13) without control. Here the parameters of the rotor and bearing are chosen as $S_{o k}=S_{o} \frac{\Omega}{\omega}=\frac{F_{\text {stat }} \psi^{2}}{2 W R \eta_{\text {oil }} \omega}=0.390625, \omega=5001 / \mathrm{s}$, $\mu=0.87, \varepsilon=0.05 \mathrm{~mm}$, while $\gamma_{i k}$ and $\beta_{i k}(i, k=z, \nu)$ are taken from Gasch and Pfuetzner (1975). Figure 6 reveals that the amplitude will grow to infinity, if the operational speed reaches the stability margin, say $\Omega_{\lim } \approx 7001 / s$. 


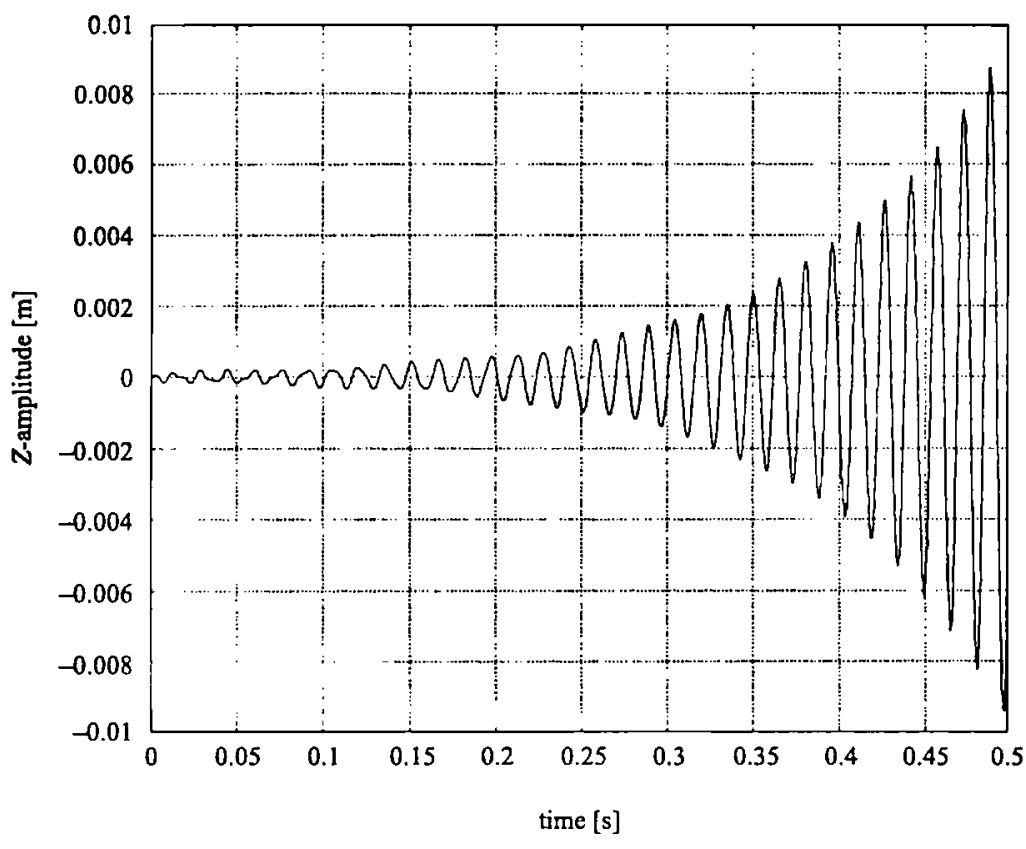

a

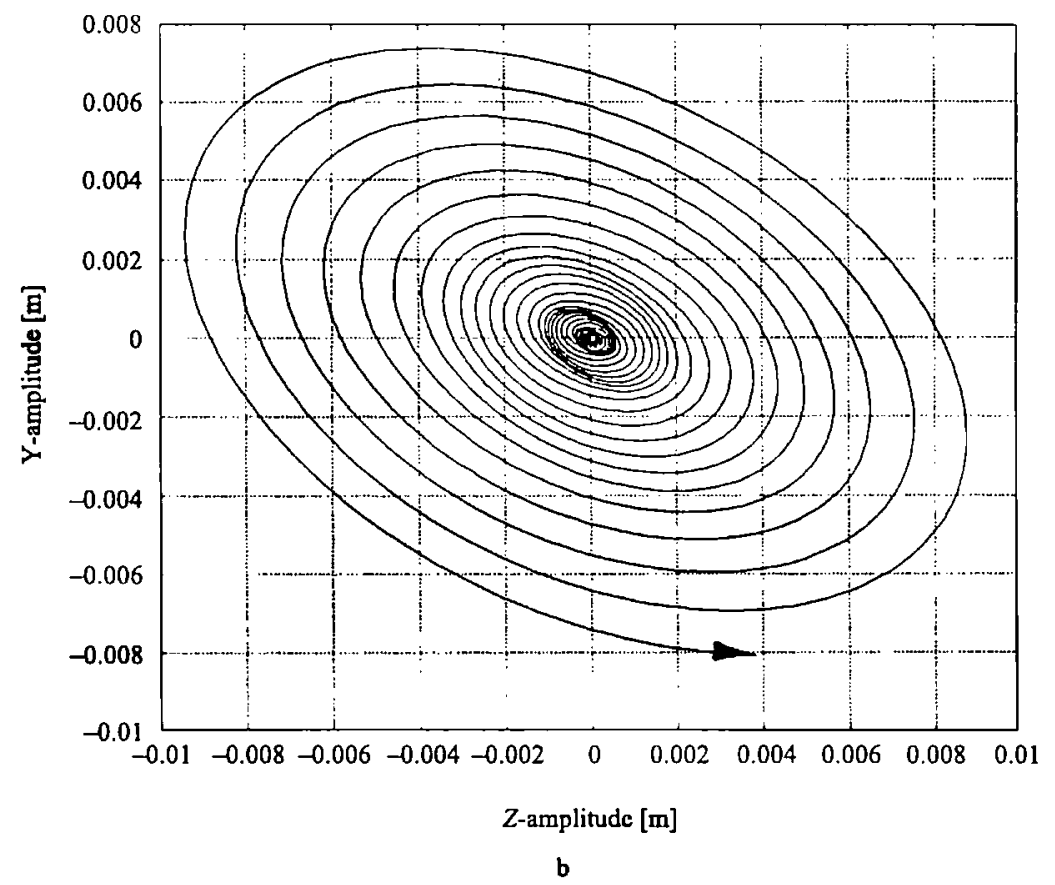

Figure 7. The unstable motion of a rotor supported by journal bearings at an operational speed over the stability margin. (a) The vibration in direction $z$; (b) the orbit of the rotor. 
Table 2. Relationship of the control parameters $a$ and $b$ to the real and imaginary parts, $R$ and $U$, of the dominant root.

\begin{tabular}{cccc}
\hline$a$ & $b$ & $R$ & $U$ \\
\hline-0.01 & -0.2 & -67.73 & \pm 368.32 \\
-0.03 & -0.1 & -35.78 & \pm 413.34 \\
-0.03 & -0.2 & -77.28 & \pm 378.52 \\
-0.03 & -0.3 & -97.82 & \pm 329.03 \\
-0.04 & -0.2 & -82.00 & \pm 384.04 \\
\hline
\end{tabular}

Figures 7 (a) and (b) illustrate the unstable vibration in direction $z$ and the orbit of the rotor at an operational speed over the stability margin, that is $\Omega=7701 / \mathrm{s}$.

\subsection{Stability Improvement of Motion of the Controlled Rotor}

We return to the characteristic equation (20). The eigenvalue can be assumed to be $\lambda=$ $R+i U$. Separating the real and imaginary parts of equation (20) gives two equations for unknown variables $R, U, a_{z}, b_{z}, a_{y}$, and $b_{y}$ as follows:

$$
\begin{aligned}
& \operatorname{Re}\left(R, U, a_{z}, b_{z}, a_{y}, b_{y}\right)=0 \\
& \operatorname{Im}\left(R, U, a_{z}, b_{z}, a_{y}, b_{y}\right)=0 .
\end{aligned}
$$

For the uncontrolled rotor $a_{z}=b_{z}=a_{y}=b_{y}=0$, as case 1 the same parameters are chosen, see Figurc 6 , and the angular speed $\Omega=7701 / s$. Numerically solving equations (29) and (30) can find the values of $R$ and $U$, which satisfy both equations at the same time. Figure 8 shows a plot of the solutions to equations (29) and (30). The points where the lines intersect are the characteristic roots. It can be seen that there are two roots that have a positive real part (11.817 $\pm j 412.93)$. Hence, for case 1 the motion of the uncontrolled rotor is unstable (see Figures 7(a) and (b)).

For the controlled rotor, let $a_{z}=a_{y}$ and $b_{z}=b_{y}$, so as to reduce the number of the unknown variables. In terms of equations (29) and (30), the control parameters are searched, which makes the real part of all the characteristic roots negative.

For case 1 , we choose $a_{z}=a_{y}=-0.03$ and $b_{z}=b_{y}=-0.1$. From Figure 9 , one can see that all the characteristic roots have a negative real part. Hence, the particular solution to equation (13) is asymptotically stable. To confirm this result, equation (13) is integrated numerically. The result of this integration is shown in Figures 10 (a) and (b). Figure 10 (a) shows the vibration of the controlled rotor in direction $z$, and (b) the orbit of the rotor. It is evident that with the control parameters $a_{z}=a_{y}=-0.03$ and $b_{z}=b_{y}=-0.1$, the periodic motion of the rotor becomes stable. Table 2 give the relationship of the control parameters $a$ and $b$ to the real and imaginary parts, $R$ and $U$, of the dominant root.

Even with smaller control parameters (absolut), that is $a_{z}=a_{y}=-0.01$ and $b_{z}=h_{1}=$ -0.07 , the stability will also obviously be improved. Figure 11 shows the response amplitude of the uncontrolled and controlled rotor to imbalance versus the operational speed. It reveals that the periodic response of the rotor to imbalance in the stable range is not influenced by the control, but the stability margin is expanded from $\Omega_{\lim } 700 \mathrm{l} / \mathrm{s}$ to $895 \mathrm{l} / \mathrm{s}$, say about $28 \%$. 


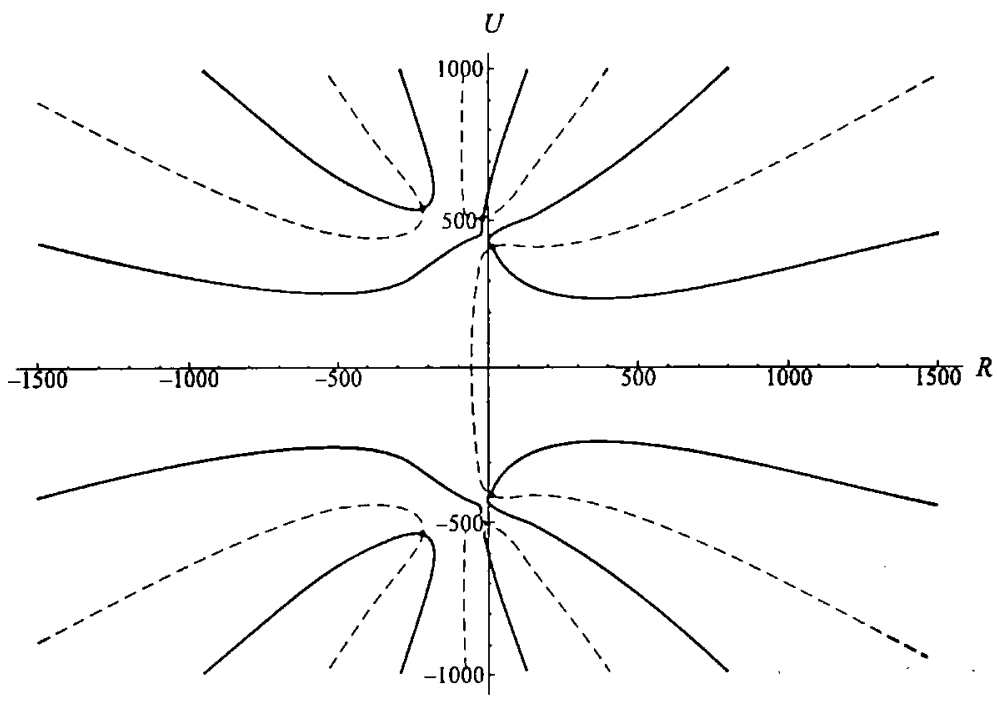

Figure 8. Real and imaginary parts of the roots of the characteristic equation (21) of the uncontrolled rotor (points where the lines intersect are the roots).

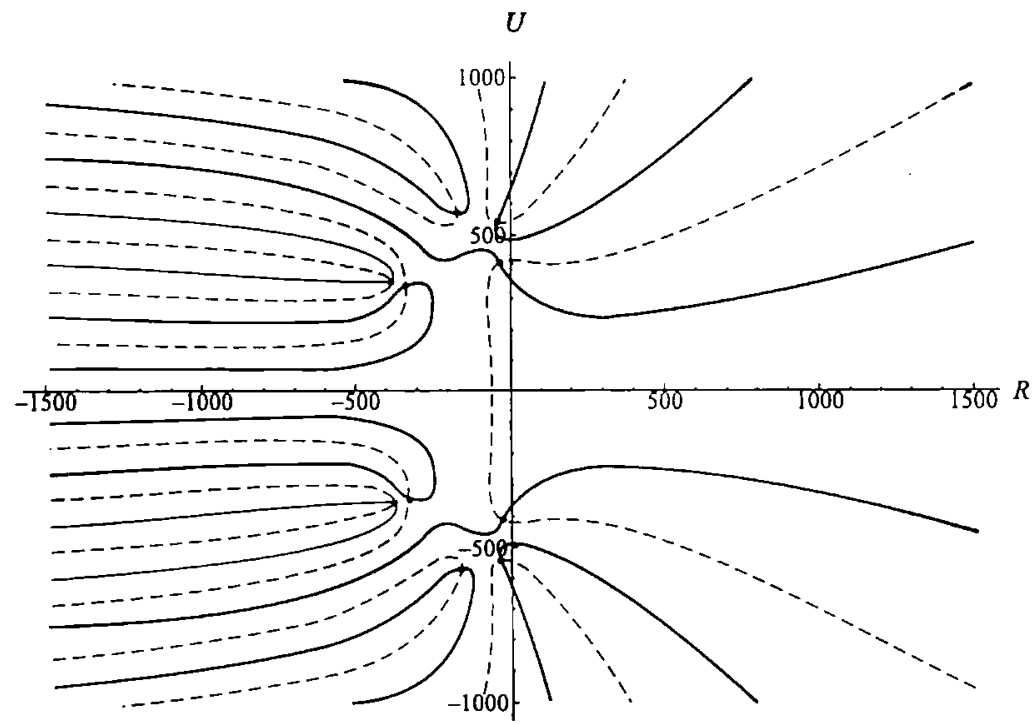

Figure 9. Real and imaginary parts of the roots of the characteristic equations (29) and (30) of the controlled rotor with $a=-0.03$ and $b=-0.1$. The solid lines are the solution to equation (29); the dashed lines are the solution to equation (30); the intersection points are the solution to equations (29) and $(30)$. 

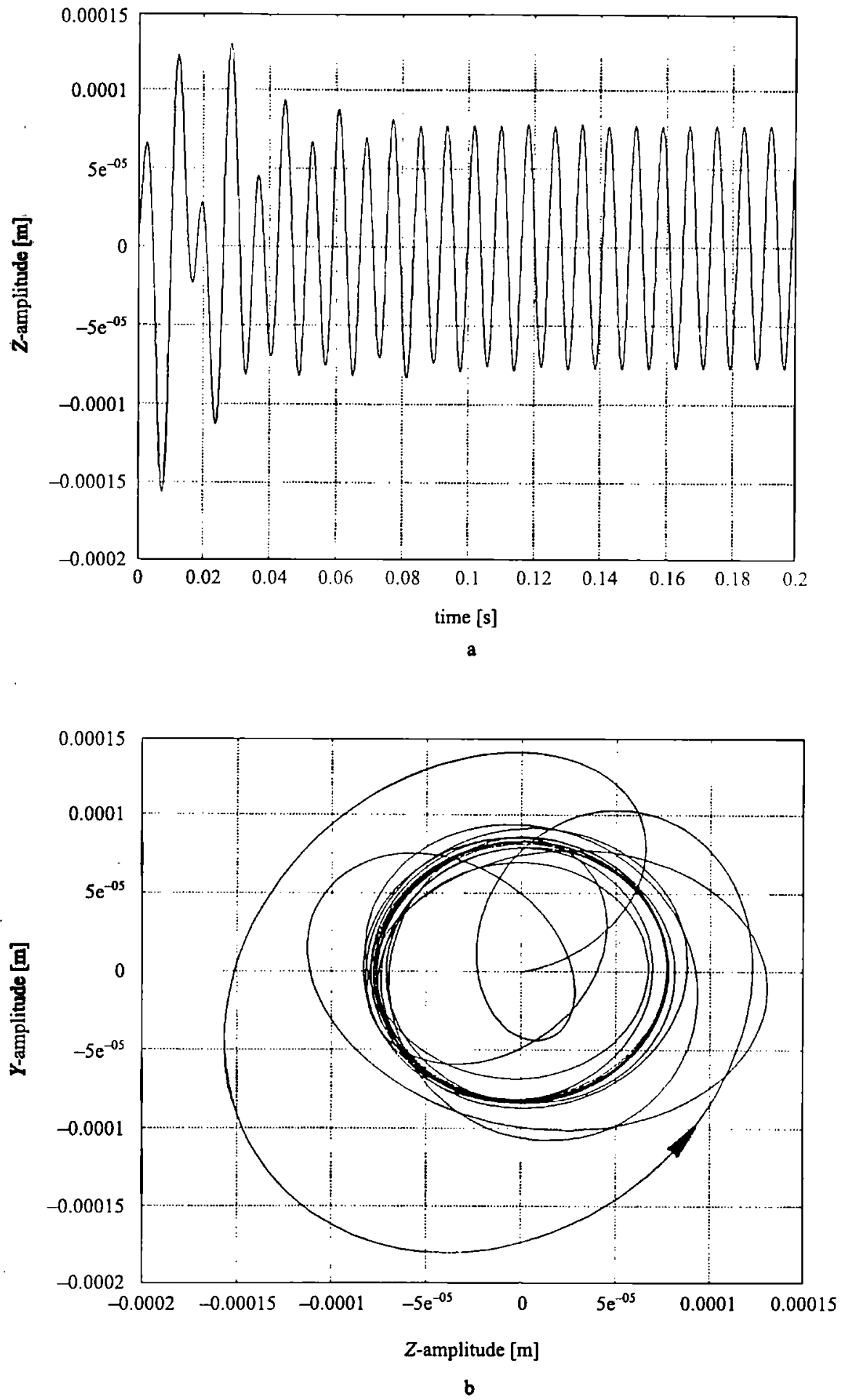

Figure 10. The stabilization of the unstable motion with parameters $a_{z}=a_{y}=-0.03$ and $b_{z}=b_{y}=-0.1$. (a) The vibration in direction $z$; (b) the orbit of the rotor. 


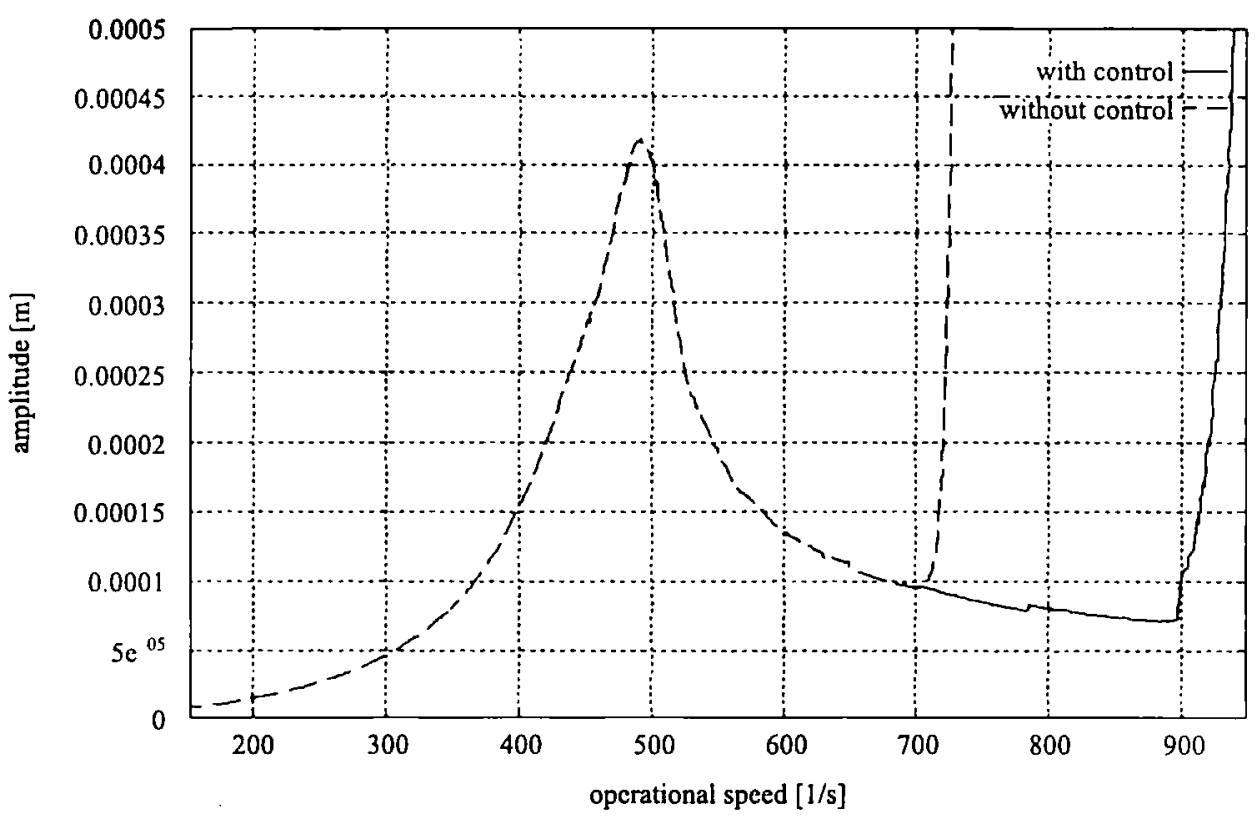

Figure 11. The response amplitude of the uncontrolled and controlled rotor to imbalance, parameters $a_{z}=a_{y}=-0.01$ and $b_{z}=b_{y}=-0.07$.

\section{CONCLUSIONS}

The phase-locked delayed feedback control method is first applied to a rotor supported by rolling-element bearings with negative damping and then to a rotor supported by joumal bearings. The control parameters are determined by numerically solving the characteristic equation of the controlled rotor system. The result shows that the control method has successfully stabilized the unstable periodic vibration due to imbalance without changing it at all. Particularly, the method has greatly expanded the stability margin of motion of a rotor supported by journal bearings, thus making the rotor much less sensitive to external disturbances.

\section{REFERENCES}

Faragher, J. S., 1996, Stability Improvement of Periodic Motion of Helicopter Rotor Blades, Ph.D. dissertation, Department of Mechanical and Manufacturing Engincering, University of Melboume, Australia.

Gasch, R. and Liao, M. F., 1996, "Stability improvement of a rotor supported in joumal bearings," ILR Mitt. 308, Institut fuer Luft- und Raumfahrt, Technical University of Berlin.

Gasch, R. and Pfuctzner, H., 1975, Rotordynamik, Eine Einfuchrung, Springer Verlag, Berlin, Heidelberg, New York.

Krodkiewski, J. M. and Faragher, J. S., 1995, "Stability improvement of the periodic motion of non-lincar systems," in Proceedings of the International Conference on Vibration and Noise, Venice.

Somcya, T., Mitsui, J., Esaki, J., Saito, S., Kanemitsu, Y, Iwatsubo, T., Tanaka, M., Hisa, S., Fujikawa, T., and Kanki, H., 1989, Journal-Bearing Databook, Springer-Verlag, Berlin, New York, Tokyo. 